REVIEW

\title{
Tuberculosis preventive therapy: An underutilised strategy to reduce individual risk of $T B$ and contribute to TB control
}

\author{
G J Churchyard, R E Chaisson, G Maartens, H Getahun \\ Gavin Churchyard is Chief Executive Officer: Aurum Institute, Johannesburg, South Africa, and an Honorary Professor in the School of Public \\ Health, University of the Witwatersrand, Johannesburg, South Africa. Richard Chaisson is Director: Center for Tuberculosis Research, Johns \\ Hopkins University School of Medicine, Baltimore, MD, USA. Gary Maartens is Professor and Chair of the Division of Clinical Pharmacology, \\ Department of Medicine, Faculty of Health Sciences, University of Cape Town, South Africa. Haileyesus Getahun is Coordinator, TB/HIV and \\ Community Engagement, Global TB Programme, World Health Organization, Geneva, Switzerland.
}

Corresponding author: G J Churchyard (gchurchyard@auruminstitute.org)

\begin{abstract}
Tuberculosis (TB) remains a global health problem, and South Africa (SA) has one of the world's worst TB epidemics. The World Health Organization (WHO) estimated in 1999 that one-third of the world's population was latently infected with TB. In SA up to $88 \%$ of HIVuninfected young adults (31 - 35 years) are latently infected with TB. In the most recent meta-analysis, 6 - 12 months of isoniazid preventive therapy (IPT) was associated with a lower incidence of active TB than placebo (relative risk (RR) 0.68 ; $95 \%$ confidence interval (CI) 0.54 0.85 ), with the greatest benefit among individuals with a positive tuberculin skin test (TST) (RR $0.38 ; 95 \%$ CI 0.25 - 0.57 ). A clinical trial of IPT given with antiretroviral therapy (ART) for 12 months reduced TB incidence by $37 \%$ compared with ART alone (hazard ratio (HR) 0.63 ; $95 \%$ CI 0.41 - 0.94). The effect of IPT is limited in high-burden countries. IPT for 36 months v. 6 months reduced TB incidence among HIVpositive, TST-positive participants by $74 \%$ (HR 0.26 ; $95 \%$ CI $0.09-0.80$ ). A study of more than 24000 goldminers confirmed that IPT is safe, with only $0.5 \%$ experiencing adverse events. A meta-analysis of studies of IPT since 1951 did not show an increased risk of developing resistance. Alternative TB preventive therapy regimens, including high-dose isoniazid and rifapentine given weekly for 3 months, have been shown to have similar efficacy to IPT. Mathematical modelling suggests that scaling up continuous IPT targeted to HIV-positive persons, when used in combination with other treatment and prevention strategies, may substantially improve TB control.
\end{abstract}

S Afr Med J 2014;104(5):339-343. DOI:10.7196/SAMJ.8290

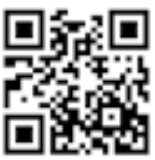

\section{Burden of tuberculosis (TB) disease}

TB remains a global health problem with an estimated burden of disease in 2012 of 8.6 million new cases, $13 \%$ co-infected with HIV, and an estimated 1.3 million TB deaths. ${ }^{[1]}$ South Africa (SA) has one of the world's worst $\mathrm{TB}$ epidemics, with the highest $\mathrm{TB}$ incidence among the 22 highest TB burden countries in the world, estimated at 933/ 100000 population in 2012, the third-largest absolute number of cases and the largest number of HIV-associated TB cases. ${ }^{[1]}$ Approximately $65 \%$ of TB patients in SA are HIV-infected. ${ }^{[1]}$

\section{Burden of latent TB infection (LTBI)}

The World Health Organization (WHO) estimated in 1999 that onethird of the world's population is latently infected with Mycobacterium tuberculosis. ${ }^{[2]}$ The greatest burden of LTBI is in South-East Asia (46\%), the Western Pacific region (32\%), Africa (31\%) and the Eastern Mediterranean region (27\%). ${ }^{[3]}$ Sub-Saharan Africa, however, has the largest number of persons with LTBI who are co-infected with HIV. In high-transmission settings such as SA, up to $88 \%$ of HIV-uninfected young adults (31 - 35 years) are latently infected with TB, and $89 \%$ of SA goldminers are TB-infected. ${ }^{[4-7]}$ These data suggest that the burden of LTBI in SA is enormous. The prevalence of tuberculin skin test (TST) positivity among people co-infected with HIV is estimated to be $22.8 \%$ overall and highest among those with $\mathrm{CD} 4^{+}$counts $\geq 500$ cells/ $\mu \mathrm{l}(37.4 \%)$. $^{[8]}$ This is explained by the compromised ability of HIV-infected persons to react to the skin test because of cutaneous anergy associated with immunosuppression.

\section{Risk of TB disease}

LTBI occurs when individuals infected with M. tuberculosis harbour the organism in a latent state, characterised by slowed or intermittent metabolism and replication below the level necessary to produce clinical illness. ${ }^{[9]}$ The lifetime risk of reactivation of latent infection in healthy HIV-uninfected individuals is $10 \%$, with $5 \%$ developing TB during the first 2 - 5 years after infection. The risk is greatly increased in the context of immunosuppression, most notably due to HIV infection. ${ }^{[3]}$ The WHO estimates that in countries with a generalised HIV epidemic, HIV-infected persons have a 20 - 37-fold greater risk of developing TB than HIV-uninfected persons. Although antiretroviral treatment (ART) reduces the risk of TB by approximately twothirds, ${ }^{[10-12]} \mathrm{TB}$ remains a common cause of morbidity ${ }^{[13,14]}$ and a leading cause of early mortality in individuals on ART. ${ }^{[15]}$

\section{Purpose of this article}

The purpose of this article is to present the evidence to support isoniazid preventive therapy (IPT) use, particularly continuous IPT (cIPT), in HIV-positive people as part of a combination of TB prevention strategies to reduce individual risk of $\mathrm{TB}$ and to contribute to $\mathrm{TB}$ control. Alternative $\mathrm{TB}$ preventive therapy regimens for 
persons living with HIV and preventive therapy for HIV-uninfected persons at high risk of TB are also discussed. The paper focuses exclusively on TB preventive therapy for adults.

\section{IPT in HIV infection \\ History of IPT in SA}

The IPT guidelines for TST-positive people living with HIV were initially incorporated into the SA ART guidelines in 2005. Uptake of IPT was poor after introduction of these guidelines, largely due to TST creating a programmatic barrier to implementation and concerns of generating isoniazid resistance. ${ }^{[16]}$

In 2010, in line with the WHO recommendations, SA revised its national IPT guidelines and removed TST as a requirement to initiate IPT, to facilitate programmatic implementation of IPT. The uptake of IPT increased dramatically and more than 375000 South Africans living with HIV were started on IPT in 2011 and 2012, respectively, making the IPT programme one of the largest in the world. Despite this, uptake of IPT among people living with HIV in care, including pregnant women, remains poor. The SA IPT guidelines were re-issued in 2013 and recommend at least 36 months of IPT for TST-positive HIV-infected persons, including people on ART; 6 months of IPT for those whose TST status is unknown, regardless of whether they are on ART or not; and 12 months of IPT for persons on ART if their TST is negative. ${ }^{[17]}$ People living with HIV who are not on and do not require ART, and who have a negative TST, do not need IPT.

\section{Screening for TB}

Screening for active TB disease is required before commencing IPT to minimise the risk of developing drug resistance by inadvertently treating active TB with an inadequate regimen. An individual participant metaanalysis showed that a symptom screen of a cough (any duration), night sweats, loss of weight and fever was able to identify HIV-positive people with a very low probability of having undiagnosed active TB disease. ${ }^{[18]}$ Chest radiography combined with symptom screening increased the sensitivity of TB screening, particularly among SA goldminers. ${ }^{[18-20]}$ On the basis of these results, the $\mathrm{WHO}$ recommends that TB disease among people living with HIV be excluded before starting IPT, using a symptom screen of current cough, night sweats, fever and weight loss. ${ }^{[21]}$ In HIV clinics that routinely screen their patients at every clinic visit, the WHO TB symptom screen performs less well, particularly among patients on ART. ${ }^{[22]}$ In settings with a high prevalence of undiagnosed TB disease, some experts recommend excluding TB by doing a sputum culture or, in sick hospitalised patients, using a lateral flow assay for mycobacterial lipoarabinomannan on urine combined with Xpert MTB/RIF on sputum or urine. ${ }^{[22-24]} \mathrm{TB}$ screening before starting IPT may detect TB cases earlier, thereby reducing transmission and TB-associated mortality.

\section{Efficacy of 6 - 12 months of IPT}

In the most recent meta-analysis of people with HIV, 6 or 12 months of IPT was associated with a $32 \%$ lower incidence of active TB than placebo (relative risk (RR) 0.68 ; 95\% confidence interval (CI) 0.54 $0.85){ }^{[25]}$ This benefit was greatest among individuals with a positive TST (RR 0.38; 95\% CI 0.25 - 0.57). ${ }^{[25]}$ IPT was also effective when implemented in a routine HIV care programme for SA goldminers, prior to ART availability ${ }^{[26]}$ IPT, given for $6-12$ months after TB treatment, is effective in reducing the risk of recurrent $\mathrm{TB}$ disease and is recommended by the WHO. ${ }^{[21,27]}$

\section{IPT with ART}

IPT combined with ART reduced the risk of TB disease by $80-97 \%$ and death by up to $50 \%$ in HIV-infected persons. ${ }^{[28-31]} \mathrm{A}$ recent individually randomised, pragmatic, controlled trial demonstrated that
IPT, taken for 12 months with ART, decreased TB incidence by $37 \%$ (hazard ratio (HR) 0.63 ; 95\% CI 0.41 - 0.94) overall compared with ART alone. ${ }^{[32,33]}$ The effectiveness of IPT was similar when assessed by interferon-gamma release assay and TST status. ${ }^{[33]}$ The results of this study underpin the current SA guidelines for IPT with ART.

\section{cIPT}

Recent evidence from studies conducted in high-transmission settings suggests that cIPT may be beneficial. Among HIV-infected participants in Botswana who received 36 v. 6 months of IPT, TB incidence was reduced by $43 \%$ overall (HR $0.57 ; 95 \%$ CI $0.33-0.99$ ); among TST-positive participants TB incidence was reduced by $74 \%$ (HR 0.26; 95\% CI 0.09 - 0.80), whereas TST-negative participants received no benefit (HR 0.75; 95\% CI $0.38-1.46) .{ }^{[31]}$ In the per protocol analysis, there were no incident cases of TB among TSTpositive persons who took 36 months of IPT. Among TST-positive, HIV-infected SA adults, incidence rates of TB or death were similar between participants who received CIPT or 6 months of IPT in the intention-to-treat analysis ( 2.7 v. 3.6/100 person-years, incidence rate ratio (IRR) 0.75 ; $95 \%$ CI $0.38-1.38){ }^{[34]}$ In the per protocol analysis, the incidence of TB or death was reduced by $58 \%$ in the cIPT study arm, compared with the 6 months of IPT arm.

\section{Durability of IPT}

In the pre-HIV era, IPT was associated with a durable reduction in TB incidence in mental institutions and Alaskan villages. ${ }^{[35-38]}$ In the HIV pre-ART era, 6 months of IPT in high-transmission settings was durable for up to 18 months. ${ }^{[39,40]}$ In the ART era, IPT has limited durability in high TB transmission settings. ${ }^{[40,41]}$ In the Botswana trial of $6 \mathrm{v}$. 36 months of IPT, among persons who took 6 months of IPT the risk of TB increased approximately 200 days after stopping IPT, ${ }^{[31]}$ while among persons who took 36 months of IPT, TB incidence increased by $90 \%$ after stopping IPT ${ }^{[42]}$ Among SA goldminers, the incidence of TB in the intention-to-treat analysis was reduced by $58 \%$ during the intended 9-month IPT period. TB incidence increased rapidly following cessation of IPT and was similar to that observed for those who did not start IPT in the post-treatment period. ${ }^{[43]}$

\section{Safety of IPT}

The safety of IPT was recently confirmed in more than 23500 SA goldminers. With clinical monitoring during the 9 months of IPT, the risk of isoniazid-associated adverse events, including clinical hepatitis, was low $(0.5 \%)$, even among older patients and those on ART. ${ }^{[44]}$ In the individually randomised trial of IPT with ART v. placebo with ART, participants on IPT were 2.13 times (95\% CI 0.97 - 4.67) more likely to discontinue the study medication due to grade 3 adverse events or raised liver enzymes. ${ }^{[32]}$ Among HIV-positive adults in Botswana, the risk of severe adverse events and death was similar in the 6-month and cIPT groups; however, the risk of death among persons with a negative TST was increased, although none of the deaths were attributed to isoniazid. ${ }^{[31]}$ Among HIV-infected South Africans taking cIPT (v. 6 months of IPT), serious adverse reactions were more common (18.4 v. 15.4/100 person-years) as was permanent discontinuation of treatment $(36.5 \%$ v. $1.9 \%) .{ }^{[34]}$ Grade 3 or 4 elevation of aspartate or alanine aminotransferase during the treatment phase was much more common with cIPT than with 6 months of IPT (28.0\% v. $5.5 \%$; $p<0.001) .{ }^{[34]}$

\section{Isoniazid resistance}

Theoretically, if active TB is missed and the bacterial load is large enough, treatment with monotherapy or an inadequate regimen has the potential to generate drug resistance. A systematic review of 
studies of IPT conducted between 1951 and 2005 concluded that IPT was not associated with an increased risk of isoniazid resistance and that isoniazid resistance is much more likely to result from inadequate treatment of active disease. ${ }^{[45]}$ The studies of cIPT in Botswana and SA did not show an increased risk of isoniazid resistance. ${ }^{[31,34]}$ Among 126 goldminers with TB after starting IPT, the prevalence of isoniazid resistance and treatment outcomes were similar to those in TB cases without previous exposure to IPT. ${ }^{[46]}$ The impact of wide-scale uptake of preventive therapy on generating drug resistance is unknown. Mathematical modelling suggests that the emergence of isoniazid resistance with IPT use may be reduced by delivering IPT to those who will benefit the most and by improved case finding and prompt treatment of those with drug-resistant TB disease. ${ }^{[47,48]}$

\section{Population-level effect of IPT}

In the pre-HIV era, community-wide IPT was evaluated in Alaska, Greenland and Tunisia, all of which had epidemic TB. In Alaska, IPT was given to households for 1 year, which reduced the risk of TB by $69 \% \cdot{ }^{[35]}$ As the intervention was so successful, 12 months of IPT was given to both placebo and IPT groups 5 years later, and the population-level effect has been durable for more than 3 decades. ${ }^{[36]}$ In Greenland and Tunisia, the effect of community-wide IPT was modest (31\% and $26 \%$ reduction in TB incidence, respectively), largely owing to an inadequate dose of isoniazid and/or poor adherence to IPT in both of the trials. ${ }^{[49,50]}$ The Thibela TB study ${ }^{[51]}$ evaluated the effectiveness of community-wide IPT in addition to standard of care, compared with standard of care alone, among SA goldminers. The intervention, which is unprecedented in the history of TB control, included mass screening for TB using chest radiography and symptoms, simultaneously linked to treatment for active disease or latent infection. Despite reducing the individual risk of TB by $58 \%$ while participants took the 9 -month course of IPT, the effect waned soon after stopping IPT and there was no measurable reduction in TB incidence or prevalence at a population level after the intervention was completed ${ }^{[43]}$ Mathematical modelling suggested that even if the intervention was optimally implemented, a $20 \%$ reduction in TB incidence at a population level was the most that could be expected. ${ }^{[51]}$ The modelling also suggested that cIPT would have a profound impact on TB incidence at a population level. ${ }^{[51]}$

\section{TB after IPT: Possible mechanisms}

TB that occurs after IPT may be due to reactivation of inadequately treated TB or reinfection with a new strain of TB. Isoniazid impairs mycobacterial wall synthesis and is a potent bactericidal drug that results in a rapid decline in actively multiplying mycobacteria. A recent study showed that isoniazid rapidly killed actively dividing M. smegmatus. However, a few cells continued to divide in the presence of isoniazid owing to dynamic persistence associated with stochastic pulsing of catalase-peroxidase (KatG), which activates isoniazid. ${ }^{[52]}$ When isoniazid was withdrawn, the surviving cells underwent exponential growth again and were susceptible to isoniazid when it was reintroduced, suggesting that they had not developed resistance to the drug. It is likely that a similar phenomenon occurs when M. tuberculosis is treated with isoniazid. In support of this hypothesis, mathematical modelling of the Thibela TB intervention was not able to mirror the observed data unless the model assumed that IPT does not cure LTBI. If the model is correct and IPT does not cure LTBI, reactivation of inadequately treated LTBI after stopping treatment may in part explain the lack of durable benefit. In high-transmission settings, reinfection with a new strain of TB has been shown to be an important cause of recurrent $\mathrm{TB}$ disease after successful completion of treatment in HIV-infected and HIV-uninfected TB patients. ${ }^{[3,54]}$ In settings with a high annual risk of infection such as SA (estimated at $4.7 \%$ per year), it is likely that reinfection will contribute to the limited durability of IPT. In high-burden settings, both reactivation of inadequately treated LTBI and reinfection are likely to limit the durability of IPT. Regardless of the mechanism of TB after IPT, cIPT will reduce the risk of TB in persons living with HIV. ${ }^{[31,42]}$

\section{Alternative TB preventive therapy regimens \\ Efficacy of alternative preventive therapy regimens}

Alternative TB preventive therapy regimens have also been evaluated in people living with HIV. A meta-analysis of TB preventive therapy regimens found rifampicin-containing regimens (rifampicin and pyrazinamide; isoniazid and rifampicin; isoniazid, rifampicin and pyrazinamide) not to be more effective than IPT alone. ${ }^{[25]}$ In the pre-ART era, however, the rifampicin-based regimens were shown to have a more durable effect than isoniazid alone. ${ }^{[40,41]}$

A novel regimen of intermittent high-dose isoniazid and rifapentine given weekly for 3 months has recently been evaluated in low and high TB burden settings. Rifapentine is a rifamycin with a long half-life and greater potency against $M$. tuberculosis than rifampicin. In mouse models of LTBI, rifapentine had a greater sterilising effect than isoniazid, ${ }^{[55]}$ and weekly high-dose rifapentine and isoniazid for 3 months (3HP) had greater efficacy than isoniazid alone. ${ }^{[56]}$ The TB Trials Consortium Study $26^{[57]}$ compared 3 months of high-dose once-weekly isoniazid $(900 \mathrm{mg})$ and rifapentine (900 mg) (3HP) with 9 months of daily isoniazid (300 mg) (9H) in a non-inferiority study design.The study was conducted in low to medium TB and HIV burden settings (USA, Canada, Brazil and Spain). Eligible participants aged 2 years and above were enrolled into the study, regardless of HIV status. The study showed that $3 \mathrm{HP}$ was non-inferior to $9 \mathrm{H}$ in the modified intention-to-treat and per protocol analysis, and there was a trend towards superiority in reducing TB incidence (9H: 16/100 person-years; 3HP: 7/100). The $3 \mathrm{HP}$ study arm had a higher treatment completion rate than the $9 \mathrm{H}$ arm $(82 \%$ v. $69 \%) \cdot{ }^{[57]}$ As few HIV-infected persons were enrolled into Study 26 (2.6\% HIV-infected), an ancillary study was conducted to evaluate safety, tolerability and efficacy of $3 \mathrm{HP}$ in HIV-infected adults. Among 399 HIV-infected persons (193 9H, 206 3HP), 3HP was non-inferior to $9 \mathrm{H}$, with cumulative TB incidences in the $3 \mathrm{HP}$ v. the $9 \mathrm{H}$ arm of 1.01 and $3.69 / 100$ person-years, respectively. ${ }^{[8,59]} 3 \mathrm{HP}$ compared with $9 \mathrm{H}$ in $\mathrm{HIV}$-infected persons was tolerable and had higher completion rates $(88 \%$ v. $64 \%)$. ${ }^{[5,59]}$

The $3 \mathrm{HP}$ regimen was also compared with 6 months of IPT $(6 \mathrm{H})$ in HIV-infected, ART-naive (at enrolment), TST-positive SA adults. In the intention-to-treat analysis, the incidence rates of active $\mathrm{TB}$ or death in the $3 \mathrm{HP}$ and $6 \mathrm{H}$ arms were similar (3.1 v. 3.6/100 personyears, respectively, crude IRR 0.87 (95\% CI 0.54 - 1.39)). ${ }^{[34]}$ Based on the results of TB Trials Consortium Study 26, the US Centers for Disease Control (CDC) issued recommendations for the use of the 3HP regimen for the treatment of LTBI. ${ }^{[4]}$ The CDC is currently evaluating self-administered, as opposed to directly observed, $3 \mathrm{HP}$.

\section{Safety of alternative preventive therapy regimens}

Rifampicin-based regimens (rifampicin and pyrazinamide; isoniazid and rifampicin; isoniazid, rifampicin and pyrazinamide), compared with IPT, in people with HIV are associated with a greater rate of treatment discontinuation due to adverse effects. ${ }^{[25]}$ In Study 26 participants, the vast majority of whom were HIV-uninfected, there was reduced drug-related hepatotoxicity in the $3 \mathrm{HP}$ study arm 
compared with the $9 \mathrm{H}$ arm $(0.4 \%$ v. $2.7 \%)$, but rates of permanent discontinuation due to an adverse event were higher $\left(4.9 \%\right.$ v. 3.7\%). ${ }^{[57]}$ In the follow-on trial to Study 26 among HIV-infected persons taking $3 \mathrm{HP}$ v. $9 \mathrm{H}$, the rates of drug discontinuation due to adverse drug reactions were similar (3.4\% v. 4.2\%). ${ }^{[58,59]}$ Among HIV-infected, TSTpositive adult South Africans taking $3 \mathrm{HP}$ v. 6 months of IPT, grade 3 or 4 hepatotoxicity (elevated alanine or aspartame aminotransferase) occurred less commonly in the $3 \mathrm{HP}$ study arm $(1.5 \%$ v. $5.5 \%$, respectively), and the rates of permanent discontinuation of the study drug were similar (1.8\% v. $1.9 \%$, respectively). ${ }^{[34]}$

\section{TB preventive therapy for HIV- uninfected persons at high risk of TB}

TB preventive therapy is recommended for HIV-uninfected persons who are receiving chemotherapy or anti-tumour necrosis factor (TNF) therapy and are at increased risk of developing TB. The South African Rheumatism and Arthritis Association recommends a regimen of either isoniazid or rifampicin for 3 months or IPT for 6 - 9 months for TST-positive persons prior to starting anti-TNF drugs. Silicosis is an occupational lung disease that results from chronic silica dust exposure and is associated with an approximately threefold greater risk of TB than in silica-exposed workers who do not have silicosis. The National Department of Health has included recommendations for HIV-uninfected persons with silicosis in the 2013 IPT guidelines: 36 months of IPT if TST-positive; 6 months IPT if TST unknown; no IPT if TST-negative. For people living with HIV and silicosis, the IPT guidelines for people living with HIV should be followed.

\section{Combination TB prevention: The role of IPT}

The WHO recommends IPT for people living with HIV as part of a combination of $\mathrm{TB}$ prevention strategies that include infection control to reduce exposure to $\mathrm{TB}$, intensified case finding to detect cases earlier, and ART to reduce vulnerability to TB, in addition to reducing the numbers lost to follow-up prior to starting $\mathrm{TB}$ treatment and reducing the time to starting TB treatment. ${ }^{[1,60-62]}$ However, implementation of these strategies has been poor. Mathematical modelling has suggested that high coverage of IPT, particularly if it is given continuously and in combination with other treatment and prevention strategies, will contribute to $\mathrm{TB}$ control and accelerate progress towards elimination of the disease. ${ }^{[1,63,64]}$

\section{Conclusion}

IPT is safe, does not generate isoniazid resistance, and reduces the risk of TB among persons living with HIV, particularly if given continuously to those with evidence of TB infection. Continuous IPT should be used in combination with other TB treatment and prevention strategies to contribute to TB control. Alternative TB preventive therapy regimens may be considered for certain patients at high risk of TB.

\footnotetext{
1. World Health Organization. Global Tuberculosis Report 2013. WHO/HTM/TB/2013.11. Geneva: WHO, 2012 .

2. Dye C, Scheele S, Dolin P, Pathania V, Raviglione MC. Consensus statement. Global burden of tuberculosis: Estimated incidence, prevalence, and mortality by country. WHO Global Surveillance and Monitoring Project. JAMA 1999;282(7):677-686. [http://dx.doi.org/10.1001/jama.282.7.677]

3. Corbett EL, Watt CJ, Walker N, et al. The growing burden of tuberculosis: Global trends and interactions with the HIV epidemic. Arch Intern Med 2003;163(9):1009-1021. [http://dx.doi.org/10.1001/archinte.163.9.1009] 4. Gomez JE, McKinney JD. M. tuberculosis persistence, latency, and drug tolerance. Tuberculosis (Edinb) 2004;84(1-2):29-44. [http://dx.doi.org/10.1016/j.tube.2003.08.003]

5. Young DB, Gideon HP, Wilkinson RJ. Eliminating latent tuberculosis. Trends Microbiol 2009;17(5):183-188. [http://dx.doi.org/10.1016/j.tim.2009.02.005]

6. Wood $\mathrm{R}$, Liang $\mathrm{H}, \mathrm{Wu} \mathrm{H}$, et al. Changing prevalence of tuberculosis infection with increasing age in highburden townships in South Africa. Int J Tuberc Lung Dis 2010;14(4):406-412.

7. Hanifa Y, Grant AD, Lewis J, Corbett EL, Fielding K, Churchyard G. Prevalence of latent tuberculosis infection among gold miners in South Africa. Int J Tuberc Lung Dis 2009;Jan13(1):39-46.
}

8. Kerkhoff AD, Kranzer K, Samandari T, et al. Systematic review of TST responses in people living with HIV in under-resourced settings: Implications for isoniazid preventive therapy. PLoS One 2012;7(11):e49928. [http:// dx.doi.org/10.1371/journal.pone.0049928]

9. Manabe YC, Bishai WR. Latent Mycobacterium tuberculosis - persistence, patience, and winning by waiting Nat Med 2000;6(12):1327-1329. [http://dx.doi.org/10.1038/82139]

10. Badri M, Wilson D, Wood R. Effect of highly active antiretroviral therapy on incidence of tuberculosis in South Africa: A cohort study. Lancet 2002;359(9323):2059-2064. [http://dx.doi.org/10.1016/S0140-6736(02)089043]

11. Lawn SD, Churchyard G. Epidemiology of HIV-associated tuberculosis. Curr Opin HIV AIDS 2009;4(4):325 333. [http://dx.doi.org/10.1097/COH.0b013e32832c7d61]

12. Lawn SD, Wood R, De Cock KM, Kranzer K, Lewis JJ, Churchyard GJ. Antiretrovirals and isoniazid preventive therapy in the prevention of HIV-associated tuberculosis in settings with limited health-care resources. Lancet Infect Dis 2010;10(7):489-498. [http://dx.doi.org/10.1016/S1473-3099(10)70078-5]

13. Saraceni V, King BS, Cavalcante SC, et al. Tuberculosis as primary cause of death among AIDS cases in Rio de Janeiro, Brazil. Int J Tuberc Lung Dis 2008;12(7):769-772.

14. Lawn SD, Myer L, Orrell C, Bekker LG, Wood R. Early mortality among adults accessing a community-based antiretroviral service in South Africa: Implications for programme design. AIDS 2005;19(18):2141-2148. [http://dx.doi.org/10.1097/01.aids.0000194802.89540.el]

15. Lawn SD, Harries AD, Anglaret X, Myer L, Wood R. Early mortality among adults accessing antiretrovira Lawn SD, Harries AD, Anglaret X, Myer L, Wood R. Early mortality among adults accessing antiretroviral
treatment programmes in sub-Saharan Africa. AIDS 2008;22(15):1897-1908. [http://dx.doi.org/10.1097/ QAD.0b013e32830007cd]

16. Bristow CC, Larson E, Vilakazi-Nhlapo AK, Wilson M, Klausner JD. Scale-up of isoniazid preventive therapy in PEPFAR-assisted clinical sites in South Africa. Int J Tuberc Lung Dis 2012;16(8):1020-1022. [http://dx.doi org/10.5588/ijtld.11.0744]

17. South African Department of Health. The South African Antiretroviral Treatment Guidelines 2013. http:// www sanac org za/resources/cat_view/1-resources 2014 (accessed 31 March 2014).

18. Getahun H, Kittikraisak W, Heilig CM, et al. Development of a standardized screening rule for tuberculosis in people living with HIV in resource-constrained settings: Individual participant data meta-analysis of observational studies. PLoS Med 2011;8(1):e1000391. [http://dx.doi.org/10.1371/journal.pmed.1000391]

19. Day JH, Charalambous S, Fielding KL, Hayes RJ, Churchyard GJ, Grant AD. Screening for tuberculosis prio to isoniazid preventive therapy among HIV-infected gold miners in South Africa. Int J Tuberc Lung Dis 2006;10(5):523-529.

20. Lewis JJ, Charalambous S, Day JH, et al. HIV infection does not affect active case finding of tuberculosis in South African gold miners. Am J Respir Crit Care Med 2009;180(12):1271-1278. [http://dx.doi.org/10.1164/ rccm.200806-846OC]

21. World Health Organization. Guidelines for intensified tuberculosis case-finding and isoniazid preventive therapy for people living with HIV in resource-constrained settings. 2011. http://whqlibdoc who int publications/2011/9789241500708_eng pdf (accessed 24 March 2014).

22. Rangaka MX, Wilkinson RJ, Glynn JR, et al. Effect of antiretroviral therapy on the diagnostic accuracy of symptom screening for intensified tuberculosis case finding in a South African HIV clinic. Clin Infect Dis 2012;55(12):1698-1706. [http://dx.doi.org/10.1093/cid/cis775]

23. Shah M, Ssengooba W, Armstrong D, et al. Comparative performance of urinary lipoarabinomannan assays and Xpert MTB/RIF in HIV-infected individuals with suspected tuberculosis in Uganda. AIDS 2014. [Epub ahead of print] [http://dx.doi.org/10.1097/QAD.0000000000000264]

24. Lawn SD, Kerkhof A, Bourton R, et al. Massive diagnostic yield of HIV associated tuberculosis using rapid urine assays in South Africa. Conference on Retroviruses and Opportunistic Infections, 3-6 March 2014, Boston, MA, USA. Abstract 811LB.

25. Akolo C, Adetifa I, Shepperd S, Volmink J. Treatment of latent tuberculosis infection in HIV infected persons. Cochrane Database of Systematic Reviews 2010, Issue 1. Art. No.: CD000171. Cochrane Database Syst Rev 2010; CD000171. [http://dx.doi.org/10.1002/14651858.CD000171.pub3]

26. Grant $\mathrm{AD}$, Charalambous $\mathrm{S}$, Fielding KL, et al. Effect of routine isoniazid preventive therapy on tuberculosis Grant $\mathrm{AD}$, Charalambous S, Fielding KL, et al. Effect of routine isoniazid preventive therapy on tuberculosis
incidence among HIV-infected men in South Africa: A novel randomized incremental recruitment study incidence among HIV-infected men in South Africa: A novel randomized in
JAMA 2005;293(22):2719-2725. [http://dx.doi.org/10.1001/jama.293.22.2719]

27. Churchyard GJ, Scano F, Grant AD, Chaisson RE. Tuberculosis preventive therapy in the era of HIV infection Overview and research priorities. J Infect Dis 2007;196(Suppl 1):S52-S62. [http://dx.doi.org/10.1086/518662]

28. Golub JE, Pronyk P, Mohapi L, et al. Isoniazid preventive therapy, HAART and tuberculosis risk in HIVinfected adults in South Africa: A prospective cohort. AIDS 2009;23(5):631-636. [http://dx.doi.org/10.1097/ QAD.0b013e328327964f]

29. Golub JE, Saraceni V, Cavalcante SC, et al. The impact of antiretroviral therapy and isoniazid preventive therapy on tuberculosis incidence in HIV-infected patients in Rio de Janeiro, Brazil. AIDS 2007;21(11):14411448. [http://dx.doi.org/10.1097/QAD.0b013e328216f441]

30. Charalambous S, Grant AD, Innes C, et al. Association of isoniazid preventive therapy with lower early mortality in individuals on antiretroviral therapy in a workplace programme. AIDS 2010;24(Suppl 5):S5-S13. [http://dx.doi.org/10.1097/01.aids.0000391010.02774.6f]

31. Samandari T, Agizew TB, Nyirenda S, et al. 6-month versus 36-month isoniazid preventive treatment for tuberculosis in adults with HIV infection in Botswana: A randomised, double-blind, placebo-controlled trial. Lancet 2011:377(9777):1588-1598.

32. Rangaka MX, Boulle A, Wilkinson RJ, et al. Randomized controlled trial of isoniazid preventive therapy in HIV-infected persons on antiretroviral therapy. 19th International AIDS Conference, 22-27 July 2012 Washington, DC, USA. Abstract THLBB 03.

33. Rangaka MX, Wilkinson R, Wilkinson K, et al. Effect of tuberculin skin testing or interferon-release on the benefit of concurrent isoniazid preventive therapy with ART: Subgroup analysis of a randomized controlled trial. 20th Conference on Retroviruses and Opportunistic Infections, 3-6 March 2013, Atlanta, GA, USA Poster abstract 189LB

44. Martinson NA, Barnes GL, Moulton LH, et al. New regimens to prevent tuberculosis in adults with HIV infection. N Engl J Med 2011;365(1):11-20. [http://dx.doi.org/10.1056/NEJMoa1005136]

35. Comstock GW, Ferebee SH, Hammes LM. A controlled trial of community-wide isoniazid prophylaxis in Alaska. Am Rev Respir Dis 1967;95(6):935-943. [http://dx.doi.org/10.1056/NEJMoa1005136]

36. Comstock GW, Baum C, Snider DE Jr. Isoniazid prophylaxis among Alaskan Eskimos: A final report of the Bethel isoniazid studies. Am Rev Respir Dis 1979;119(5):827-830.

37. Ferebee SH, Hopwood L, Palmer CE. Studies on prevention of experimental tuberculosis with isoniazid. II . Ferebee $\mathrm{SH}$, Hopwood L, Palmer CE. Studies on prevention of experim
Effects of different dosage regimens. Am Rev Tuberc 1956;74(6):917-939.

Effects of different dosage regimens. Am Rev Tuberc 1956;74(6):917-939.
38. Ferebee SH, Mount FW, Murray FJ, Livesay VT. A controlled trial of isoniazid prophylaxis in mental instutions. Am Rev Respir Dis 1963;88:161-175.

Am Rev Respir Dis 1963;88:161-175.
39. Johnson JL, Okwera A, Hom DL, et al. Duration of efficacy of treatment of latent tuberculosis infection in HIV-infected adults. AIDS 2001;15(16):2137-2147. [http://dx.doi.org/10.1097/00002030-200111090-00009]

40. Quigley MA, Mwinga A, Hosp M, et al. Long-term effect of preventive therapy for tuberculosis in a cohor of HIV-infected Zambian adults. AIDS 2001;15(2):215-222. [http://dx.doi.org/10.1097/00002030-20010126000011]

1. Whalen CC, Johnson JL, Okwera A, et al. A trial of three regimens to prevent tuberculosis in Ugandan adults infected with the human immunodeficiency virus. Uganda-Case Western Reserve University Research Collaboration. N Engl J Med 1997;337(12):801-808. [http://dx.doi.org/10.1056/NEJM199709183371201]

42. Samandari T, Agizew T, Tedla Z, et al. TB incidence increase after cessation of 36 months isoniazid prophylaxis in HIV+ adults in Botswana 19th Conference on Retroviruses and Opportunistic Infections, 5-8 March 2012 Seattle, WA, USA. Abstract 147

43. Churchyard GJ, Fielding KL, Lewis JJ, et al. A trial of mass isoniazid preventive therapy for tuberculosis control. N Engl J Med 2014;370(4):301-310. [http://dx.doi.org/10.1056/NEJMoa1214289] 
44. Grant AD, Mngadi KT, van Halsema CL, Luttig MM, Fielding KL, Churchyard GJ. Adverse events with isoniazid preventive therapy: Experience from a large trial. AIDS 2010;24(Suppl 5):S29-S36. [http://dx.doi org/10.1097/01.aids.0000391019.10661.66]

45. Balcells ME, Thomas SL, Godfrey-Faussett P, Grant AD. Isoniazid preventive therapy and risk for resistant tuberculosis. Emerg Infect Dis 2006;12(5):744-751. [http://dx.doi.org/10.3201/eid1205.050681]

46. van Halsema CL, Fielding KL, Chihota VN, et al. Tuberculosis outcomes and drug susceptibility in individuals exposed to isoniazid preventive therapy in a high HIV prevalence setting. AIDS 2010;24(7):1051-1055. [http:// dx.doi.org/10.1097/QAD.0b013e32833849df

47. Mills HL, Cohen T, Colijn C. Response to comment on 'Community-wide isoniazid preventive therapy drives drug-resistant tuberculosis: A model-based analysis'. Sci Transl Med 2013;5(204):204lr4.

48. Mills HL, Cohen T, Colijn C. Community-wide isoniazid preventive therapy drives drug-resistant tuberculosis A model-based analysis. Sci Transl Med 2013;5(180):180ra49.

9. Horwitz O, Payne PG, Wilbek E. Epidemiological basis of tuberculosis eradication. 4. The isoniazid trial in Greenland. Bull World Health Organ 1966:35(4):509-526.

50. Nyboe J, Farah AR, Christensen OW. Report on tuberculosis chemotherapy pilot project (Tunisia 9). WHO/ TB/Technical Information/10. 22 April 1963

51. Chihota VN, Popane F, Churchyard GJ, et al. Community-wide isoniazid preventive therapy among gold miners in South Africa: The Thibela TB study. South African TB Conference 2012, $12-15$ June 2012, Durban, South Africa. http://www.auruminstitute.org/thibela-TB/Thibela-SA-TB-conf-12-06-12.pdf (accessed 11 April 2014).

52. Wakamoto Y, Dhar N, Chait R, et al. Dynamic persistence of antibiotic-stressed mycobacteria. Science 2013;339(6115):91-95. [http://dx.doi.org/10.1126/science.1229858]

53. Verver S, Warren RM, Beyers N, et al. Rate of reinfection tuberculosis after successful treatment is highe than rate of new tuberculosis. Am J Respir Crit Care Med 2005;171(12):1430-1435. [http://dx.doi.org/10.1164/ rccm.200409-1200OC

54. Charalambous S, Grant AD, Moloi V, et al. Contribution of reinfection to recurrent tuberculosis in South African gold miners. Int J Tuberc Lung Dis 2008;12(8):942-948.

5. Miyazaki E, Chaisson RE, Bishai WR. Analysis of rifapentine for preventive therapy in the Cornell mouse model of latent tuberculosis. Antimicrob Agents Chemother 1999:43(9):2126-2130.
56. Chapuis L, Ji B, Truffot-Pernot C, O’Brien RJ, Raviglione MC, Grosset JH. Preventive therapy of tuberculosis with rifapentine in immunocompetent and nude mice. Am J Respir Crit Care Med 1994;150(5 Part 1):13551362. [http://dx.doi.org/10.1164/ajrccm.150.5.7952564]

57. Sterling TR, Villarino ME, Borisov AS, et al. Three months of rifapentine and isoniazid for latent tuberculosis infection. N Engl J Med 2011;365(23):2155-2166. [http://dx.doi.org/10.1056/NEJMoal104875]

58. Sterling TR, Person AK. TB Trials Consortium Study 26.AIDS Clinical Trials Group 5259. Tolerability among HIV-infected persons of three months of once-weekly rifapentine + INH (3HP) vs. 9 months of daily INH $(9 H)$ for treatment of latent tuberculosis infection. The PREVENT TB Study. 19th International AIDS Conference, $22-27$ July 2012, Washington, DC, USA. Abstract MOAB0302.

59. Sterling T, Benson C, Scott N, et al. Three months of weekly rifapentine+INH for M. tuberculosis infection in HIV-infected persons. Conference on Retroviruses and Opportunistic Infections, 3-6 March 2014, Boston, MA, USA. Abstract 817

60. World Health Organization. The Stop TB Strategy: Building on and enhancing DOTS to meet the TB-related Millenium Development Goals. http://whqlibdoc who int/hq/2006/WHO_HTM_STB_2006 368 eng pdf (accessed 24 March 2014)

61. World Health Organization. WHO policy on collaborative TB/HIV activities: Guidelines for national programmes and other stakeholders. http://www who int/tb/publications/2012/tb_hiv_ policy $9789241503006 /$ en/index html (accessed 24 March 2014)

62. Suthar AB, Lawn SD, Del Amo J, et al. Antiretroviral therapy for prevention of tuberculosis in adults with HIV: A systematic review and meta-analysis. PLoS Med 2012;9(7):e1001270. [http://dx.doi.org/10.1371/journal.
pand

63. Dye C, Glaziou P, Floyd K, Raviglione M. Prospects for tuberculosis elimination. Annu Rev Public Health 2013;34(7):271-286. [http://dx.doi.org/10.1146/annurev-publhealth-031912-114431]

64. Abu-Raddad LJ, Sabatelli L, Achterberg JT, et al. Epidemiological benefits of more-effective tuberculosis vaccines, drugs, and diagnostics. Proc Natl Acad Sci U S A 2009;106(33):13980-13985. [http://dx.doi. org/10.1073/pnas.0901720106]

Accepted 6 April 2014

\section{This month in the $S A M J$}

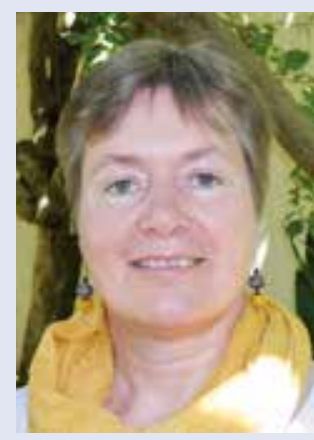

Jenny Edge, , guest editor for CME this month, is a general surgeon with a particular interest in the management of breast diseases. She completed her undergraduate training at University College Hospital, London, also undertaking an intercalated BSc in Anthropology and graduating BSc, MBBS. She went on to pursue an FRCS and MMed (Surg), undertaking her surgical training at Stellenbosch University. She worked in the UK and New Zealand before joining her husband in South Africa. She now works at the Christiaan Barnard Memorial Hospital and is an honorary lecturer at the University of Cape Town.

*Edge J. Pink ribbons for breast cancer awareness - a perspective. S Afr Med J 2014;104(5):321. [http://dx.doi.org/10.7196/SAMJ.8300]

Edge J. Breast Cancer. S Afr Med J 2014;104(5):376. [http://dx.doi.org/10.7196/SAMJ.8262]

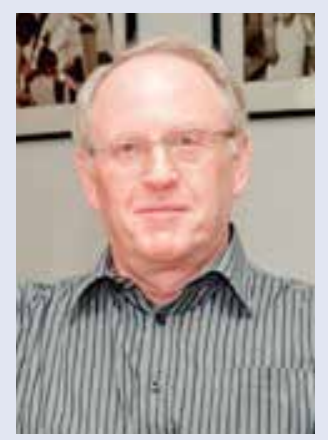

Gavin Churchyard ${ }^{\star}$ is a specialist physician and founder and Chief Executive Officer of the Aurum Institute, an independent, not-for-profit, proudly South African public benefit organisation that focuses on tuberculosis and HIV service delivery, management and research. He is an honorary professor at the School of Public Health, University of the Witwatersrand, Johannesburg, and at the Faculty of Epidemiology and Population Health, Department of Infectious Diseases, London School of Hygiene and Tropical Medicine. Prof. Churchyard is Chair of the World Health Organization (WHO) Task Force for the Development of New Policies for the Treatment of TB and a member of the WHO Strategic Technical Advisory Group for TB. He is Vice-Chair of the NIH/DAIDS-funded AIDS Clinical Trials Group (ACTG) Network and the Transformative Science Group for TB, and Chair of the ACTG Host Directed Therapy for TB Working Group.

${ }^{*}$ Churchyard GJ, Chaisson RE, Maartens G, Getahun H. Tuberculosis preventive therapy: An underutilised strategy to reduce individual risk of TB and contribute to TB control. S Afr Med J 2014;104(5):339-343. [http://dx.doi.org/10.7196/SAMJ.8290] 